\title{
Principales causas biomecánicas de la Lumbalgia Mecánica en Deportistas y posible intervención fisioterapéutica basada en ejercicios terapéuticos
}

\author{
Main biomechanical causes of Mechanical Back Pain in Athletes and possible physiotherapeutic \\ intervention based on therapeutic exercises \\ Gwendolyne Arce Salazar ${ }^{1}$, Juan Rivera Vargas ${ }^{2} \square$
}

Filiación:

1.Terapéuta Físico, MSc, Escuela de Terapia Física, Universidad Santa Paula, Curridabat, Costa Rica. $\square$

Correspondencia:

Gwendolyne Arce

garce@uspsantapaula.com

Filiación:

2. Terapéuta Físico, MsC. Clínica Santa Paula, Universidad Santa Paula, Curridabat, Costa Rica.

Correspondencia:

Juan Rivera Vargas

juanmiriva@hotmail.com

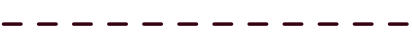

Forma de citar: Arce Salazar G, Rivera Vargas J. Principales causas biomecánicas de la lumbalgia mecánica en deportistas y posible intervención fisioterapéutica basada en ejercicios terapéuticos. Rev Ter. 2017;11(2):7-14

Declaración:El artículo es parte de una tesis para obtener el grado de maestría en Terapia Física con mención en rehabilitación deportiva

Financiamiento:

Autofinanciado

Conflictos de Interés:

El autor declara no tener conflictos de interés en la presente publicación

\section{Resumen}

Objetivo: proponer una intervención fisioterapéutica basada en ejercicios terapéuticos de activación y fortalecimiento de la unidad central o CORE. Materiales y métodos: Se realizó una revisión bibliográfica en revistas científicas indexadas ubicada en bases datos como Ebsco Host, SclELO y Medline, publicados en inglés y español durante el periodo 2006-2017, utilizando como descriptores de búsqueda dolor lumbar, ejercicios terapéuticos y estabilidad espinal. De los 42 artículos encontrados, se incluyeron 22 artículos científicos clasificados según el nivel de evidencia de Sackett como sigue: 2 artículos de nivel 1 (9\%), 6 de nivel 2 (27\%), 6 de nivel $3(27 \%), 2$ de nivel 4 (9\%) y 6 de nivel 5 (27\%). Resultados: La lumbalgia mecánica o inespecífica es una de las lesiones más comunes en los deportistas, se puede dar por causas multifactoriales como las neuromusculares, por debilidad de patrones de las cadenas musculares, inestabilidad articular, disminución en la movilidad de rangos articulares, entre otras. Además, producen cambios posturales dinámicos y estáticos alterando la biomecánica de la columna lumbar, pelvis y cadera. Conclusiones Los ejercicios terapéuticos deben ir enfocados a activar y fortalecer las unidades del CORE para contrarrestar la inestabilidad y el desequilibrio muscular, mediante un protocolo de trabajo de las cadenas musculares favoreciendo el equilibrio y mejorar las posturas dinámicas durante el gesto deportivo.

Palabras Clave: Dolor en la región lumbar, ejercicios de estiramiento muscular, fisioterapia (Fuente: DeCS-BIREME)

\section{Abstract}

Objective: to propose a physiotherapeutic intervention based on therapeutic exercises of activation and strengthening of the central unit or CORE. Materials and methods: A bibliographic review was made in indexed scientific journals located in databases such as Ebsco Host, SclELO and Medline, published in English and Spanish during the 20062017 period, using lumbar pain, therapeutic exercises and spinal stability as search terms. Of the 42 articles found, 22 scientific articles classified according to Sackett's level of evidence were included as follows: 2 articles of level $1(9 \%), 6$ of level $2(27 \%)$, 6 of level $3(27 \%), 2$ of level $4(9 \%)$ and 6 of level $5(27 \%)$. Results: Mechanical or 
nonspecific lumbago is one of the most common injuries in athletes, it can be due to multifactorial causes such as neuromuscular causes, weakness of muscle chain patterns, joint instability, decreased range of joint mobility, among others. In addition, they produce dynamic and static postural changes altering the biomechanics of the lumbar, pelvic and hip spine. Conclusions: The therapeutic exercises should be focused on activating and strengthening the CORE units to counteract the instability and muscle imbalance, by means of a working protocol of the muscular chains favoring the balance and improving the dynamic postures during the sporting gesture.

Key words: Low Back Pain, Muscle Stretching Exercises, Physical Therapy Specialty (Source: MeSH-NLM)

\section{Introducción}

Debido al aumento de práctica de diferentes deportes a nivel competitivo, que obliga a desarrollar y perfeccionar las capacidades físicas, las cuáles mejoran con más horas de entrenamiento y cambios en el estilo de vida, pueden venir acompañadas de lesiones de tipo traumáticas o micro traumática, también llamadas lesiones por sobre uso que constituyen un porcentaje elevado de las lesiones causadas por el gesto deportivo. La tasa de lesiones deportivas en la población en general es de 15,4 cada 1.000 personas de las cuales del 30 a $50 \%$ son lesiones micro traumáticas ${ }^{1}$.

Las lesiones por sobre uso son las más frecuentes en la actividad física y equivalen entre un 30\% a $50 \%$ de todas las lesiones deportivas, afectan $20 \%$ a $30 \%$ de los deportistas y son menos frecuentes en las mujeres en donde se presentan en un $15 \%{ }^{2}$.

La lumbalgia mecánica (LM), llamada también no especifica, es una patología generada por sobre uso, donde se presenta una nula activación del CORE, el CORE es lo que se conoce como núcleo central o parte central en tronco ${ }^{3}$. Si el CORE no se activa durante los cambios posturales y se acompaña de desequilibrio muscular y poca movilidad articular que puede cambiar la biomecánica en la realización del gesto deportivo. Es considerada una de las lesiones más comunes en el deporte, su frecuencia oscila entre un $1 \%$ hasta el $30 \%$ y son influenciados por el tipo de deporte, sexo, intensidad y la frecuencia de entrenamiento y la técnica ${ }^{4}$. Prens ${ }^{4}$ determina que en jugadores de futbol, levantadores de pesas y gimnastas, entre el 50 y $85 \%$ han sufrido en algún momento dolor lumbar bajo en la práctica deportiva profesional.

El objetivo de esta revisión fue analizar las causas biomecánicas que generan lumbalgia mecánica en deportistas y las posibles intervenciones fisioterapéuticas basadas en los ejercicios terapéutico para evitar las bajas en los deportistas.

\section{Metodología}

Se realizó una revisión bibliográfica de artículos científicos disponibles en texto completo, publicados en idiomas inglés y español, en el periodo 20062017, indexados en bases datos como Ebsco Host, ScIELO y Medline, relacionados con las lesiones deportivas más frecuentes, lumbalgia mecánica o inespecífica en deportistas o atletas de diferentes deportes, estabilidad espinal y CORE. Se utilizaron los siguientes descriptores para el algoritmo de búsqueda en ambos idiomas: Dolor lumbar, ejercicios terapéuticos, estabilidad espinal.

De la recopilación de información se tuvo un total de 42 artículos de los cuales solo se tomaron en cuenta 22 , relacionados con los objetivos de la búsqueda. 7 de ellos relacionados a intervención fisioterapéutica mediante ejercicios terapéuticos de la lumbalgia en deportistas y los restantes 15 están relacionados a la caracterización de la lumbalgia mecánica en deportistas.

Los artículos de la revisión fueron clasificados según 
el nivel de evidencia de Sackett. ${ }^{5}$

Esta sistematización permitió clasificar en 5 niveles, siendo 1, evidencia científica importante considerada como la mejor evidencia, con resultados más significativos, y siendo 5 evidencia menos buena (Tabla 1).

\section{Resultados}

La lumbalgia mecánica es una afección muy frecuente en deportistas, afecta a un número importante de atletas disminuyendo el rendimiento deportivo en pretemporada y temporadas de competición, los resultados más significativos de sus causas biomecánicas se observan en la Tabla 2.

Las causas de la lumbalgia mecánica o inespecífica en deportistas se deben al déficit de la activación de los músculos del CORE principalmente de los músculos locales como el transverso abdominal y el multifido, además de la respuesta de activación retardada en los cambios de postura que genera cambios posicionales produciendo inestabilidad la columna lumbar ${ }^{10}$.

La unidad central o CORE fueron descritos por Bergmark, de ahí grandes investigadores como McGuill y Cholewiski, así como Panjabi, han establecido aspectos importantes de la anatomía y funcionamiento de la estabilidad espinal ${ }^{16}$. Desde el punto de vista anatómico y biomecánico el CORE es una unidad de rigidez pasiva proporcionada por los ligamentos y huesos, la rigidez activa que es producto de la acción de los músculos ${ }^{17}$.

La estabilidad espinal, la define Heredia et al $^{18}$ como: "el proceso dinámico de controlar la posición estática en el contexto funcional, pero permitiendo que el tronco se mueva bajo control en otras situaciones". Hodges es considerado uno de los primeros investigadores de la estabilidad central ${ }^{18}$ y menciona aspectos importantes como el proceso dinámico, para controlar la estabilidad mientras el tronco se mueve, esto quiere decir, que durante la movilización de estructuras anatómicas en un gesto deportivo se
Tabla 1. Número de artícullos científicos incluídos en la revisión según nivel de evidencia de Sackett.

\begin{tabular}{ccc}
\hline Nivel de evidencia & Número de artículos & Porcentaje \\
\hline $\mathbf{1}$ & 2 & $9 \%$ \\
$\mathbf{3}$ & 6 & $27 \%$ \\
$\mathbf{4}$ & 6 & $27 \%$ \\
$\mathbf{5}$ & 2 & $9 \%$ \\
Total & 6 & $27 \%$ \\
\end{tabular}

Fuente de elaboración propia.

debe lograr la activación de las unidades para lograr dicha estabilidad y evitar disfunciones de la zona de CORE que a largo plazo puede llevar a generar desequilibrios con dolor mecánicos y desencadenar una lumbalgia no especifica.

En los atletas con lumbalgia mecánica la importancia de la movilidad de cadera es relevante para darle estabilidad lumbo-sacra y evitar la hipermovilidad de la columna lumbar, así mismo al fortalecer los extensores de cadera tomando en cuenta el glúteo mayor como motor principal evita la inhibición generada por la activación e hipertonicidad del olio-psoas, logrando que la pelvis mantenga su alineación normal y facilite el trabajo de activación y fortalecimiento de los músculos de CORE que generen fuerza de compresión en la columna lumbar durante los cambios activos de postura es decir durante el gesto deportivo.

Igualmente $\mathrm{Panjabi}^{16}$ menciona que la importancia de la unidad neuromuscular como el que se anticipa de manera certera para activar los músculos estabilizadores durante el movimiento. Así mismo Abenhaim citado por Behm ${ }^{19}$ explica que el entrenamiento específico de los músculos estabilizadores del CORE es una consideración importante para actividades de la vida cotidiana, para el rendimiento deportivo y la rehabilitación del dolor lumbar.

Un atleta con dolor lumbar o lumbalgia mecánica cambia la biomecánica de los movimientos durante el gesto deportivo lo que aumenta la probabilidad de 
Tabla 2. Resultados principales de artículos científicos seleccionados para dolor lumbar bajo (DLB) en deportistas.

\begin{tabular}{lll}
\hline Autor (año) & Tipo de estudio y población & Resultados y Conclusiones del estudio \\
\hline
\end{tabular}

Estudio caso-control

91 hombres atletas de alto rendimiento y

Jonasson et al ${ }^{6} \quad 12$ no atletas (grupo control) con dolor

(2011) lumbar y otras articulaciones como hombro, codos, cadera, rodillas.

$\begin{array}{cl} & \\ & \text { Estudio de prevalencias } \\ \text { prospectivo. } & \\ & 272 \text { adolescentes de } 31 \text { deportes. } 158 \\ \text { Schmidt et } \text { al. }^{7} & \begin{array}{l}\text { hombres y } 113 \text { mujeres entre los } 12-20 \\ \text { años. Los deportistas entrenan entre 13 y } \\ \text { (2014) }\end{array} \\ & \begin{array}{l}5 \text { horas semanales y se observó la } \\ \text { prevalencia de DLB durante un año. }\end{array}\end{array}$
deportes relacionados con la rotación de

Harris-Hayes, et tronco.

$\mathrm{al}^{9} \quad$ Grupo sin historia de dolor lumbar que no (2009) participan regularmente en deportes que implique rotación de columna pero que son activos.

Sin historia de dolor pero que participan en deportes que impliquen rotación.

Fort $V$ et al11
(2013) $\quad$ Revisión bibliográfica

Caso- control

16 mujeres con un promedio de 19 años de edad que practican gimnasia, natación y baloncesto. 8 de ellas presentan DLB

Ashmen K et al ${ }^{12}$ con 6 meses de evolución, los 8 restantes (1996) un grupo control conocido sano. Se excluyeron las lumbalgias producto asociadas a escoliosis, trastornos neurológicos, discrepancias de los MMII, Espondilolistesis y espondilosis.

\section{Kiesel K et al $^{13}$ 2005 \\ Criterio de un experto}

Caso- control

44 atletas elite entre los 16-33 años, con

Oyarzoa $C$ et al ${ }^{14}$ dolor lumbar durante el 2011;

(2014) 20 con DLB (11 hombres y 9 mujeres) y

25 atletas $\sin$ DLB (15 hombres y 9

mujeres), miembros de los equipos

nacionales escogidos aleatoriamente.

Caso-Control

Se trabajó con dos grupos, Un grupo caso de 24 atletas ( 16 hombres y 8 mujeres) con dolo lumbar no específico que practican varios deportes. Que tienen un Shenoy $\mathbf{S} \quad$ mínimo de tres años de practicar el et al ${ }^{15}$ deporte y que entrenen un mínimo de 5

(2013)
La prevalencia de dolor lumbar fue de 36-50\%. Se encontró una correlación entre el dolor lumbar y el dolor de cadera y rodilla. Además, un aumento del dolor en atletas de alto rendimiento en la columna lumbar y otras durante el gesto deportivo. Se asocia el dolor lumbar bajo a microtrauma a repetición.

Se les realizó un cuestionario semanal y examen neurológico de sensibilidad, dermatomas, fuerza muscular máxima según Janda, pruebas de dolor y movilidad lumbar. Edad promedio de aparición del DLB fue a los 13 años. En el $31 \%$ los episodios de dolor son recurrentes y el $39 \%$ reportó dolor constante. De todos los atletas solo el 7,7\% tomaba AINES. En 38 atletas el dolor fue sin irradiación a MMII.

La prevalencia de dolor en mujeres fue de $58,4 \%$ y $66,4 \%$ en hombres. Cabe destacar que los jugadores de voleibol mostraron una prevalencia mayor de $74,3 \%$ en comparación con biatletas en un $45.7 \%$.

En atletas la prevalencia es mayor $50-70 \%$ que en personas no atletas.

Se cree que la explicación del aumento del DLB en el voleibol es el gesto deportivo por cargas axiales e hiperextensión de columna que puede generar microtraumatimos en la placa ósea.

El estudio se enfocó en características claves de la población, con dolor lumbar que regularmente realiza deporte relacionado con giros o rotaciones de la columna vertebral a nivel recreativo, que practique al menos 2 veces por semana e informa aumento de dolor durante 0 después del gesto deportivo, comparado con atletas sin historia de dolor lumbar que no sean regulares en deportes que impliquen rotación, y deportistas son dolor lumbar que si participen en esos deportes que implique rotación.

Se midió la rotación de la cadera en los grupos con flexión de rodilla a $90^{\circ}$. En el primer grupo no hubo cambios, en el segundo grupo de sujetos con dolor lumbar hubo una pequeña diferencia en la rotación y entre los MMII derecho e izquierdo. Se demostró que los pacientes con DLB presentan menos movilidad en la cadera.

EI DLB está relacionada a la falta de coordinación lumbopelvica que se asocia al momento del movimiento sobre todo al inicio del movimiento de los MMII, el dolor aumenta por un aumento del estrés del tejido, además se observó en los individuos con DLB una ante versión pélvica durante el movimiento de cadera.

A pesar de esto los resultados no son heterogéneos, el DLB se da por un aumento del estrés en el tejido sin darle chance que este se adapte.

factores relacionados con el control del Sistema Neuromuscular para DLB: Fatiga Muscular, Activación de la musculatura, Alteración de la coactivación muscular, Desequilibrios musculares, Déficit Central Postural, Disminución de la propiocepción, Déficit en el CORE y Disminución de mecanismos de anticipación.

Se realizaron 3 pruebas para valorar el nivel de DLB, utilizando la prueba de Mcgill y de flexibilidad entre ambos grupos.

El rendimiento abdominal no se encontró cambios significativos entre los dos grupos cuando se realizó la prueba de flexibilidad. Cuando se realizó la prueba de McGill Pain el grupo de caso experimento dolor leve a moderado, con el grupo control no se reportó dolor. Se atribuye el dolor a la fatiga muscular que reduce el control muscular además del desequilibrio muscular que se genera en la pelvis cambiando la postura, generando mala postura. Se encontró déficit en la flexión lateral derecha, lo que no genera una acción de la fascia toraco lumbar. Se encontró una disminución de la extensión de la cadera lo que sugiere una tensión de los flexores de cadera. Centrarse en el aumento de fuerza y flexión muscular que establece y mueven pelvis para estabilizar la misma.

La autora propone tres evaluaciones funcionales que evidencian que la no activación y poca fuerza de las Musculatura del CORE, y los cambios que produce a nivel postural que en repeticiones continuas como pasa en atletas genera micro lesiones y dolor lumbar bajo inespecífico.

En las pruebas realizadas, se hicieron en una plataforma computarizada donde cada atleta descalzo se le indicó verbalmente que debía ponerse de pie y luego es sometido a diferentes posiciones, en diferentes superficies con los ojos cerrados, primero con apoyo bipodal y luego apoyo monopodal.

Se determinó que los atletas con dolor lumbar presentan menos activación de cadenas musculares, durante los cambios de movimiento y además presentan compensaciones por una alteración propioceptiva.se encontró que el MTA si se activó sin embargo el Multifido estaba inhibido en sujetos con DLB.

Se recomienda que todo el programa de fisioterapia para atletas cuente con ejercicios de activación del CORE

Se midió mediante EMG la respuesta del músculo recto abdominal y erector de espina ante un movimiento brusco en los dos grupos caso y control.

Se midió desde el momento de la perturbación hasta la activación de la musculatura.

La latencia fue similar en ambos grupos, sin embargo, cuando la perturbación fue inesperada en el grupo caso se retrasa la latencia en un $27 \%$ respecto al grupo control.

Se ve una diferencia en los movimientos inesperados, dado que los pacientes con DLB tienen dificultados por para adaptarse a las perturbaciones, desarrollando alternativas que provocan dolor lumbar y desequilibrios.

Se cree que la disminución de la activación muscular y la respuesta neuromuscular provoca

dolor muscular ya que la columna se vuelve rígida como respuesta protectora y la acumulación

de esto da como resultado dolor crónico cirugía de columna.

Grupo control de 25 atletas (17 hombres y

8 mujeres) sin dolor lumbar bajo pero que practiquen el mismo deporte 
sufrir otras lesiones articulares debido al desequilibrio muscular que se presenta, aunado a esto la fatigabilidad de los MMII aumenta la probabilidad de padecer de dolor lumbar inespecífico.

Para entender las causas predisponentes a lesión o dolor lumbar de origen inespecífico se toma en cuenta factores tanto de postura activa como pasiva (Figura 1). Dentro de los factores de postura activa o cambios posicionales el dolor lumbar se le atribuye a la nula o escasa a activación de los músculos que forman el núcleo central o CORE, y la coordinación con la unidad neuromuscular que no activa la musculatura en el momento idóneo. En la postura pasiva los cambios de alineación de cuerpo generan alteraciones en cadenas musculares, debilitando algunos músculos y aumentado el tono en otros.

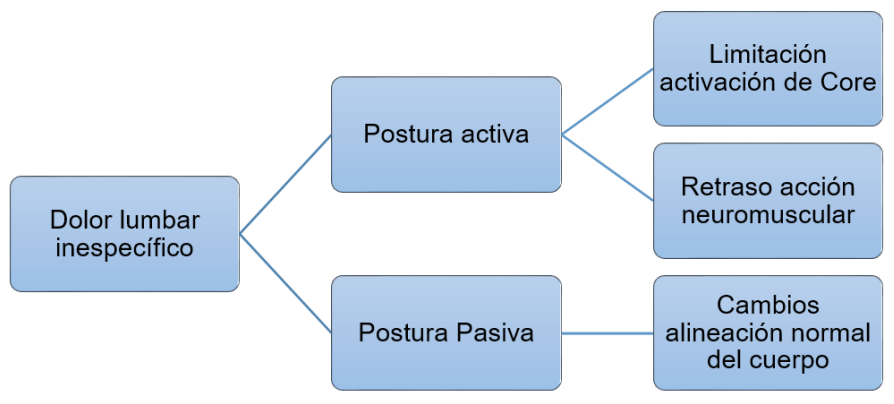

Figura 1. Factores de postura activa y pasiva en el dolor lumbar inespecífico.

Fuente: Elaboración propia, 2017.

\section{Discusión}

Los ejercicios funcionales cuentan con pilares en la realización en la prescripción de los ejercicios los cuales involucra necesariamente un ejercicio de cada uno de los siguientes patrones: empuje vertical, jalón vertical, empuje horizontal, jalón horizontal, dominante de cadera, dominante de rodilla, CORE.

Con estos pilares se asegura la participación de cadenas musculares y la estabilidad del CORE ${ }^{21}$.

Se divide en 4 etapas desarrolladas de manera progresiva para obtener mejores resultados.

- La primera etapa se evalúa de la movilidad y flexibilidad estandarizada por la Funcional
Movement Systems ${ }^{20}$ que se divide en tres pruebas fundamentales de sentadilla, movilidad de hombro y levantamiento activo de la pierna recta.

- La segunda etapa de movilidad el objetivo es mejorar y aumentar la movilidad de la cadera, tobillo y hombros, para evitar compensaciones durante el gesto deportivo.

- La tercera etapa es la de activación de las unidades musculares, contribuyendo con la estabilidad global, además de lograr estabilidad del CORE durante la actividad y realización del gesto.

- La cuarta etapa es la de fortalecimiento de las cadenas musculares para potenciar y mejorar los patrones del gesto deportivo.

Previo al inicio se realiza una etapa de calentamiento de un minuto y medio para evitar lesiones musculotendinosas. La realización de cada etapa se hace de manera progresiva, con ejercicios de los más simples a los más complejos, haciendo progresiones cada cuatro semanas aproximadamente ${ }^{20}$. Las cuatro etapas y la descripción se pueden observar en la tabla 3.

El plan de entrenamiento basado en ejercicios funcionales, trabaja de manera integral las unidades musculares, así como la activación de CORE, la recomendación es que cada atleta lo realice aparte de los entrenamiento de competición, que pueda hacerse en una sala abierta con buena ventilación y con diferentes pesos libres, barras, poleas entre otras.

El profesional a aplicarlo debe ser un terapeuta físico formado en prescripción del ejercicio, ya que es un profesional capacitado en prevención y rehabilitación de afecciones musculo esqueléticas.

El programa es tanto para rehabilitar atletas con dolor lumbar que no estén en etapas agudas, así como para prevenir el dolor lumbar mecánico en cualquier atleta de cualquier deporte.

Se cree necesario que pueda tomarse en cuenta 
Tabla 3. Intervención fisioterapéutica para el tratamiento de la lumbalgia mecánica basada en ejercicios funcionales.

\begin{tabular}{|c|c|c|}
\hline Etapa & Objetivo & Acciones \\
\hline 1. Evaluación & $\begin{array}{l}\text { Evaluar la estabilidad y la } \\
\text { movilidad de las } \\
\text { articulaciones del cuerpo. }\end{array}$ & $\begin{array}{l}\text { Evaluar mediante pruebas específicas } \\
\text { la movilidad del tobillo, la cadera, la } \\
\text { columna dorsal, y hombros. } \\
\text { Así mismo evaluar la estabilidad de la } \\
\text { rodilla, de la columna lumbar y } \\
\text { escapulas. } \\
\text { Con estas pruebas se establece de } \\
\text { manera personalizada las } \\
\text { necesidades de cada atleta en cuando } \\
\text { a movilidad y estabilidad, aclarando } \\
\text { que ambos pueden cambiar la } \\
\text { biomecánica del gesto deportivo } \\
\text { generando posibilidad de lesión por } \\
\text { micro trauma }{ }^{20}\end{array}$ \\
\hline 2. Movilidad & $\begin{array}{l}\text { Mejorar o aumentar la } \\
\text { movilidad de las } \\
\text { articulaciones móviles. }\end{array}$ & $\begin{array}{l}\text { Ejercicios específicos para cumplir con } \\
\text { el objetivo, con secuencia y frecuencia } \\
\text { específicas. }\end{array}$ \\
\hline 3. Activación & $\begin{array}{l}\text { Activar la musculatura del } \\
\text { CORE. }\end{array}$ & $\begin{array}{l}\text { Ejercicios específicos de activación del } \\
\text { CORE basados en el método Mc } \\
\text { Gill }{ }^{10} \mathrm{O} \text { escuela canadiense, } \\
\text { inicialmente con activación estática y } \\
\text { posteriormente estabilidad dinámica. }\end{array}$ \\
\hline 4. Fortalecimiento & $\begin{array}{l}\text { Fortalecer y estimular } \\
\text { propioceptivamente la } \\
\text { musculatura global } \\
\text { relacionada con la } \\
\text { estabilidad espinal. }\end{array}$ & $\begin{array}{l}\text { Ejercicios específicos enfocados al } \\
\text { fortalecimiento y estimulación de los } \\
\text { movimientos globales mejorando } \\
\text { potencia, resistencia y coordinación en } \\
\text { el gesto deportivo. }\end{array}$ \\
\hline
\end{tabular}

Fuente: Elaboración propia de los autores basado en referencias 10 y 20.

en todas las disciplinas deportivas para evitar bajas deportivas y aumentar el rendimiento de cada atleta

\section{Referencias Bibliográficas:}

1. Rodriguez C. Lumbalgias en Hockey sobre césped. [Tesis de licenciatura en Kinesiología]. Mar del Plata, AR: Universidad de Fasta; 2011. Disponible en: http://redi.ufasta.edu.ar:8080/xmlui/ bitstream/handle/123456789/324/2011_K_012. pdf? sequence $=1$
2. Marino Isaza F, Cardona Arenas O, Contreras Vergara L. Medicina del Deporte. Bogotá: Corporación para Investigaciones Biológicas; 2006.

3. Vera García FJ, Barbado D, Moreno Pérez V, Hernández Sánchez S, Juan Recio C, Elvira J. Core stability: evaluación y criterios para su entrenamiento. Rev Andal Med Deporte [Internet]. 2015;8(3):130-137. Disponible en: http://scielo. isciii.es/pdf/ramd/v8n3/revision3.pdf

4. Prens Reyes L. Prevalencia de lesiones de co- 
lumna lumbar en futbolistas profesionales en el departamento de Antioquia en el periodo 2011 - 2012 [Tesis de grado]. Medellín: Universidad CES; 2011. Disponible en: http://bdigital.ces.edu. co:8080/repositorio/bitstream/10946/1393/4/Prevalencia_lesiones_columna.pdf

5. Manterola C, Asenjo Lobos C, Otzen T. Jerarquización de la evidencia: Niveles de evidencia y grados de recomendación de uso actual. Rev Chilena Infectol [Internet]. 2014;31(6):705-718. Disponible en: https://scielo.conicyt.cl/pdf/rci/ v31n6/art11.pdf

6. Jonasson P, Halldin K, Karlsson J, Thoreson O, Hvannberg J, Baranto A, et al. Prevalence of joint-related pain in the extremities and spine in five groups of top athletes. Knee Surg Sports Traumatol Arthrosc [Internet]. 2011;19(9):1540-1546. Disponible en: http://search.ebscohost.com/login. aspx?direct=true $\& d b=r s s \& A N=64481095 \&$ lan$\mathrm{g}=$ es\&site=ehost-live

7. Schmidt C, Zwingerberger S, Walther A, Reuter $U$, Kasten P, Seifert J, et al. Prevalence of low back pain in adolescent athletes and epidemiological investigation. Int J Sports Med [Internet]. 2014;35(8):684-9. Disponible en: http://dx.doi. org/10.1055/s-0033-1358731

8. Nadler S, Malanga G, Bartoli L, Feinberg J, Prybicien M, Deprince M. Hip muscle imbalance and low back pain in athletes: influence of core strengthening. Med Sci Sports Exerc [Internet]. 2002;34(1):9-16. Disponible en: https://pdfs. semanticscholar.org/5307/3ece19d37e04ffa966ca60325405a4561c29.pdf

9. Harris Hayes M, Sahrmann S, Van Dillen L. Relationship Between the Hip and Low Back Pain in Athletes Who Participate in Rotation-Related Sports. J Sport Rehabil [Internet]. 2009;18(1):6075. Disponible en: https://www.ncbi.nlm.nih.gov/
pmc/articles/PMC2699456/pdf/nihms100498. pdf

10. McGill S. Low Back Stability: From Formal Description to Issues for Performance and Rehabilitation. Exerc Sport Sci Rev [Internet]. 2001;29(1):26-31. Disponible en: http:// www.akot.com.ar/cokiba/talleres/2015/core/files/1\%20McGill2001_LowbackStability.pdf

11. Fort Vanmeerhaeghea A, Romero Rodriguez D. Análisis de los factores de riesgo neuromusculares de las lesiones deportivas. Apunts Med Esport [Internet] 2013;48 (179):109-20. Disponible en: http://www.apunts.org/espdf-X0213371713445417

12. Ashmen K, Swanik C, Lephart S. Strength and Flexibility Characteristics of Athletes with Chronic Low-Back Pain. J Sport Rehabil [Internet]. 1996;5:275-86. Disponible en: http://www.pitt. edu/ neurolab/publications/1992-1996/AshmenKJ_1996_JSportRehab_Strength\%20 and $\% 20$ flexibility $\% 20$ characteristics $\% 20$ of $\% 20$ athletes $\% 20$ with $\% 20$ chronic $\% 20$ low-back $\% 20$ pain.pdf

13. Kiesel K, Burton L. Cook G. Mobility Screening for the Core, Part 3: Implications for Athletic Low Back Pain. Athl Ther Today [Internet]. 2005;10(1):36-9. Disponible en: http://search. ebscohost.com/login.aspx? direct=true \&db=rss\&AN=15595224\&lang=es\&site=ehost-live

14. Oyarzo C, Villagrán C,Silvestre R, Carpintero P, Berral F. Postural control and low back pain in elite athletes. Comparison of static balance in elite athletes with and without low back pain. J Back Musculoskelet Rehabil [Internet]. 2014;27(2):141-146. Disponible en: http:// search.ebscohost.com/login.aspx?direct=-

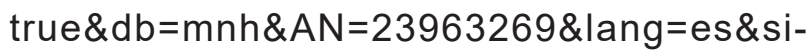
te=ehost-live 
15. Shenoy S, Balachander H, Sandhu S. Long latency reflex response of superficial trunk musculature in athletes with chronic low back pain. J Back Musculoskelet Rehabil [Internet]. 2013;26(4):445-50. Disponible en: http://search. ebscohost.com/login.aspx?direct=true $\& \mathrm{db}=\mathrm{mn}$ h\&AN=23948831\&lang=es\&site=ehost-live

16. Panjabi M. Clinical spinal instability and low back pain. J Electromyogr Kinesiol [Internet]. 2003;13(4):371-379. Disponible en: http://www. kinex.cl/papers/Lumbar/inestabilidad\%20clinica\%20panjabi\%20\%282\%29.pdf

17. Cabeza Calderón J. Variedad de planteamientos en el trabajo del Core [Tesis de grado en Ciencias de la Actividad Física y del Deporte]. León, ES: Universidad de León; 2014. Disponible en: http://buleria.unileon.es/bitstream/ handle/10612/4164/1_TFG\%20Cabeza\%20Calder\%c3\%b3n\%20Jonatan\%20\%282014\%29.pdf? sequence $=1$

18. Heredia Elvar JR, Donate FI, Mata Ordónez F, Moral S, Peña G. Revisión de los Métodos de Valoración de la Estabilidad Central (Core). PubliCE [Internet]. 2012. Disponible en: https://g-se.com/ revision-de-los-metodos-de-valoracion-de-la-estabilidad-central-core-1426-sa-g57cfb2720c148

19. Behm D, Drinkwater E, Willardson J, Drinkwater $E$, Cowley P. Declaración de posición de la Sociedad Canadiense de Fisiología del Ejercicio: La utilización de Inestabilidad para el Entrenamiento del Núcleo (CORE) en el Acondicionamiento de Poblaciones Deportivas y No Deportivas. PubliCE [Internet]. 2010. Disponible en: https://g-se. com/declaracion-de-posicion-de-la-sociedad-canadiense-de-fisiologia-del-ejercicio-la-utilizacion-de-inestabilidad-para-el-entrenamiento-del-nucleo-core-en-el-acondicionamiento-de-poblaciones-deportivas-y-no-deportivas-1430-sa-R57cfb2720c9d5
20. Sanabria M. Taller de Evaluación 2013 [CD ROM]. Curridabat: Instituto Multispa; 2013.

21. Wiatt E., Flanagan S. Lateral Trunk Flexors and Low Back Pain: Endurance and Bilateral Asymmetry. Athl Ther Today [Internet]. 2009;14(3):10-12. Disponible en: http:// search.ebscohost.com/login.aspx?direct=true $\& \mathrm{db}=\mathrm{rss} \& A N=38714485 \&$ lang $=e s \&$ site $=e-$ host-live 